\title{
Gezielte Sanktionen als Mittel der Konflikteinhegung in Afrika - Erfahrungen und Aussichten
}

\author{
Michael Brzoska*
}

\begin{abstract}
Sanctions have been frequently used as an instrument to end, or at least reduce the intensity of conflicts in Africa. Several of these have been stand-alone arms embargos. Most of the more recent sanctions, however, combine arms embargoes with other measures from the menu of "smart sanctions « such as travel restrictions and financial sanctions. Another noticeable trend in the recent past has been the use of asymmetrical sanctions by the United Nations - sanctions against one of the parties in a conflict. Not all conflicts in Subsaharan Africa have triggered sanctions. No sanctions are, for example, noted for some cases that have not been in the spotlight of international attention. In other cases, strong international partners prevented countries from being sanctioned, at least over some time. The assessment of the effectiveness depends highly on the yardstick of effectiveness. Some effects on sanctions on the targeted goods, particularly arms, can be noted in nine out of 13 sanction episodes, however, there is only one case, Angola, were the political goal seems to have been reached through sanctions.
\end{abstract}

Keywords: Afrika, Sanktionen, Konflikte, internationale Beziehungen

\section{Einführung}

$\mathrm{I}$ n keinem Kontinent der Welt war in den 1990er Jahren die Inzidenz der Kriege - ihre Zahl gemessen an der Zahl der Staaten - so hoch wie in Afrika. Während für die Welt insgesamt die Zahl der Kriege seit der Mitte der 1990er Jahre abnimmt, ist sie in Afrika bis in das 21. Jahrhundert hinein angestiegen ${ }^{2}$.

Schlimmer noch: Die Prognosen für die Zukunft Afrikas sind düster. Macht man etwa die Arbeiten der Forschungsgruppe um Paul Collier zu den Ursachen von Kriegen zur Grundlage einer Schätzung der Inzidenz von Kriegen in der Zukunft, so ist damit zu rechnen, dass auch in Zukunft Afrika der Kontinent mit den meisten Kriegen sein wird. Wesentliche Faktoren nach Collier und anderen sind niedriges Pro-Kopf-Einkommen, geringes oder gar negatives wirtschaftliches Wachstum, hohe Abhängigkeit vom Export von Rohstoffen und die Dominanz einer ethnischen Gruppe über eine oder mehrere Minderheiten ${ }^{3}$. In Untersuchungen anderer Autoren werden zum Teil andere Faktoren in den Vordergrund gestellt, wie fehlende oder marode politische Institutionen, schwache Staaten oder regionales Weitertragen von Konflikten, aber immer wieder sind die Prognosen für Afrika besonders negativ ${ }^{4}$.

Internationale Akteure haben ein breites Spektrum an Maßnahmen zur Verminderung der Zahl der Kriege und ihrer Wirkungen diskutiert, durchgeführt und analysiert, von der Rolle der Entwicklungszusammenarbeit bis hin zu militärischen Interventionen. Ein Instrument der internationalen Gemein-

* Dr. Michael Brzoska, Direktor des Instituts für Friedensforschung und Sicherheitspolitik an der Universität Hamburg. Er dankt Marc von Boemcken für die Assistenz bei der Erstellung der Daten.

2 Mikael Eriksson und Peter Wallensteen, Armed Conflict, 1989-2003, Journal of Peace Research, 41 (5), 2004.

3 Paul Collier, Lani Elliott, Håvard Hegre, Anke Hoeffler, Marta Reynal-Querol and Nicholas Sambanis, Breaking the Conflict Trap, Oxford, 2003.

4 Macartan Humphreys, Economics and Violent Conflict, Conflict Prevention Initiative, Harvard University, 2003. schaft sind Sanktionen, die Unterbrechung von Beziehungen mit einem Gegenüber, dessen Verhalten verändert werden soll.

Sanktionen rufen ein seltsam zwiespältiges Echo in der politischen und akademischen Diskussion hervor. Zum einen werden sie als sehr schwaches Instrument zur Beeinflussung des Verhaltens von "Zielen« angesehen, insbesondere in Kriegsregionen in Afrika. Die Kritik zielt dabei auf die »naive« Theorie der Sanktionen, bei der ohne viel Evidenz davon ausgegangen wird, dass die Unterbrechung von Beziehungen Entscheidungsträger dahingehend beeinflusst, ihr inkriminiertes Verhalten zu ändern ${ }^{5}$. Zum zweiten wird auf die Schwierigkeiten der Umsetzung solcher Maßnahmen in Afrika mit seinen porösen Grenzen und vielen korrupten Offiziellen hingewiesen ${ }^{6}$. Gleichzeitig wird bei aktuellen Krisen schnell nach Sanktionen gerufen, jüngstes Beispiel seit Sommer 2004 ist die Krise in Darfur im Sudan. Nicht zuletzt deutsche Regierungsvertreter, wie etwa Bundesministerin Heidemarie WieczorekZeul, machten sich zu Fürsprechern von gezielten Sanktionen gegen die Regierung in Khartum.

Es gibt mehrere Gründe für die sehr unterschiedliche Einschätzung von Sanktionen. Ein wichtiger Grund ist der Mangel an fundierten Untersuchungen der Effekte von Sanktionen. Ein weiterer, damit zusammenhängender Grund sind die sehr unterschiedlichen Erwartungen und Maßstäbe, die an Sanktionen angelegt werden. Schließlich sind Sanktionen, als eine von verschiedenen Maßnahmen die von außen zur Einhegung von Kriegen ergriffen werden können, auch immer Gegenstand politischer Auseinandersetzungen.

5 Johan Galtung, On the Effects of International Economic Sanctions: With Examples from the Case of Rhodesia, World Politics, 19 (3), S. 26-48; David Lektzian, Making Sanctions Smarter, Norwegian Committee for the Red Cross, Oslo, 2003.

6 Andrew W. Knight, The United Nations and Arms Embargo Verification, Lewiston, 1998. 
Tabelle 1: Internationale Sanktionen in Afrika seit 1990

\begin{tabular}{|c|c|c|c|}
\hline Ziel & $\begin{array}{l}\text { Internationale } \\
\text { Organisation/USA* }\end{array}$ & Sanktionstyp & Zeitraum \\
\hline Angola (UNITA) & Vereinte Nationen (VN) & $\begin{array}{l}\text { Zunächst Waffenembargo später } \\
\text { auf weitere Typen ausgeweitet }\end{array}$ & Sept. 1992-Dez. 2002 \\
\hline Côte d'Ivoire & $\begin{array}{l}\text { VN; ab Februar 2005: } \\
\text { Überwachung durch UNOCI } \\
\text { und französische Truppen }\end{array}$ & $\begin{array}{l}\text { Waffenembargo, } \\
\text { Reiseschränkungen und } \\
\text { Finanzsanktionen gegen einzelne } \\
\text { Personen }\end{array}$ & November 2004 \\
\hline Liberia & $V N$ & $\begin{array}{l}\text { Zunächst Waffenembargo, später } \\
\text { weitere Sanktionen }\end{array}$ & Nov. 1992- \\
\hline $\begin{array}{l}\text { Sierra Leone (RUF, } \\
\text { Militärregierung }(A D R C), \\
\text { Nachfolgegruppen) }\end{array}$ & $V N$ & $\begin{array}{l}\text { Waffenembargo und } \\
\text { Reisebeschränkungen, zeitweilig } \\
\text { auch Diamanten }\end{array}$ & Okt. 1997- \\
\hline $\begin{array}{l}\text { Ruanda (Regierung, später } \\
\text { Hutu-Rebellen in Rwanda } \\
\text { und Nachbarstaaten) }\end{array}$ & $V N$ & Waffenembargo & Mai 1994- \\
\hline Somalia & $V N$ & Waffenembargo & Januar 1991- \\
\hline Sudan & $\begin{array}{l}\text { Europäische Union seit } 1994 \\
\text { durchgehend, VN 1996- } \\
\text { 2001(diplomatische } \\
\text { Sanktionen) und ab Juli } 2004 \\
\text { Waffenembargo gegen } \\
\text { Janjawid }\end{array}$ & $\begin{array}{l}\text { Waffenembargo (EU, ab Juli } 2004 \\
\text { VN gegen Janjawid), ab März } \\
2005 \text { erweitert auf alle } \\
\text { Kriegsbeteiligten in Darfur und um } \\
\text { Reisebeschränkungen und } \\
\text { Finanzsanktionen gegen einzelne } \\
\text { Personen }\end{array}$ & $\begin{array}{l}\text { Mit unterschiedlichen } \\
\text { Ausprägungen seit 1994- }\end{array}$ \\
\hline
\end{tabular}

Quelle: Archiv BICC Aktive Sanktionsregime, Stand Oktober 2005

* UN-Sanktionen sind automatisch auch Sanktionen einzelner Staaten bzw. Staatengruppen.

In diesem Text soll der Beitrag von internationalen Sanktionen zur Kriegseinhegung in Afrika untersucht werden. Dabei wird folgenden Fragen nachgegangen:

- In welchen Fällen sind Sanktionen verhängt worden, in welchen nicht?

- Welche Effekte hatten die Sanktionen in den Fällen, in denen sie verhängt wurden?

- Welche Lehren lassen sich aus der Analyse der Sanktionen der Vergangenheit für die Verbesserung von Sanktionsregimen ziehen?

Mit dieser Untersuchung soll ein Beitrag zur Beantwortung der Frage geleistet werden, welche Rolle gezielte Sanktionen bei der Einhegung von kriegerischen Konflikten in der Zukunft spielen können.

\section{Sanktionen gegen Kriegsbeteiligte}

Insgesamt sind in Afrika seit 1990 gegen »Ziele« in 13 Staaten internationale Sanktionen verhängt worden, mit den Ausnahmen Libyen und $\operatorname{Togo}^{7}$ alles Kriegsbeteiligte ${ }^{8}$ (siehe Tabelle 1).

7 Die Sanktionen gegen Libyen waren Reaktionen auf terroristische Anschläge, deren Urheberschaft Libyen angelastet wurde. Die Sanktionen von ECOWAS und AU gegen Togo wurden im Februar 2005 auf Grund der nichtverfassungsgemäßen Machtübernahme nach dem Tod von Gnassigne Eyadema durch seinen Sohn Faure verhängt und nach der Ankündigung (ECOWAS) bzw. Durchführung (OAU) von Wahlen wieder aufgehoben. Damit setzten die beiden Organisationen ihre Ankündigungen durch, auf nichtdemokratische Machtwechsel mit Sanktionen zu reagieren. Schon wenige Monate später, im August 2005, blieb allerdings eine entsprechende Reaktion auf den Militärputsch in Mauretanien aus.

8 Im Falle des Sudans wurden die Sanktionen der Vereinten Nationen im Jahre 1996 wegen eines terroristischen Anschlages in Ägypten verhängt, nicht wegen der Kriegsbeteiligung der Regierung in Khartum. 
Die Zahl der »Sanktionsregime«, die sich auf Grund des Sanktionstyps, der Zielsetzungen und »Ziele« unterscheiden lassen, ist deutlich höher, da in einigen Fällen die »Ziele« mit der Übernahme (Sierra Leone) bzw. dem Verlust (Ruanda) der politischen Macht wechselten und in einer Reihe von Fällen die Sanktionstypen geändert wurden (in der Regel Ausweitung der Sanktionen).

Auffällig ist zunächst die hohe Zahl der Waffenembargos. In jedem der in Tabelle 1 aufgeführten Fälle von Sanktionen war ein Waffenembargo ein Teil der von internationalen Organisationen beschlossenen Maßnahmen (in Burundi als Teil des Handelsembargos der Nachbarstaaten). In fünf der Fälle blieb es beim Waffenembargo, ebenfalls in fünf Fällen (Liberia, Sierra Leone, UNITA in Angola, Côte d'Ivoire, Sudan) wurde das Embargo schrittweise auf weitere Bereiche ausgeweitet. Ein umfassendes Wirtschaftsembargo gab es nur in einem Fall (Burundi) und dann auch nur durch Nachbarstaaten, wenn auch mit Duldung durch die Organisation für Afrikanische Einheit (OAU).

Eine Reihe der von den Vereinten Nationen verhängten Embargos richteten sich nicht gegen alle Kriegsparteien, sondern waren »asymmetrisch «. Hierzu gehören neben den Sanktionen gegen die UNITA in Angola auch diejenigen gegen bewaffnete Gruppen im Nordosten des Kongo, gegen RUF/AFRC in Sierra Leone, gegen bewaffnete Hutu-Gruppen aus Ruanda und die im Juli 2004 beschlossenen Sanktionen gegen die Janjawid im Sudan sowie im Kongo. Die Sanktionen gegen Äthiopien/Eritrea - die einzigen im Falle eines zwischenstaatlichen Krieges und die gegen Liberia, Somalia, Ruanda, Côte d'Ivoire, und, ab März 2005, Sudan ${ }^{9}$, richteten sich gegen alle Kriegsbeteiligten. Alle eigenständigen, nicht auf VN-Beschlüssen beruhenden Sanktionen der Europäischen Union und afrikanischer Staatengruppen gegen Kriegsbeteiligte waren symmetrisch.

Die Betrachtung nur der im Oktober 2005 aktiven Sanktionsregime macht die Bedeutung asymmetrischer Sanktionsregime in der Praxis des Sicherheitsrates der Vereinten Nationen deutlich (Tabelle 1). Aktiv waren die Sanktionen gegen bewaffnete Gruppen im Kongo, aus Ruanda, in Sierra Leone und im Sudan, sowie gegen alle Akteure in Liberia, Somalia, Côte d'Ivoire und im Sudan. Drei asymmetrischen Sanktionen standen vier symmetrische gegenüber. Dies belegt den Eindruck, dass die Mitglieder des VN-Sicherheitsrats zunehmend bereit waren, kollektiv in einem Krieg Partei zu ergreifen und mit der Verhängung von Sanktionen auch die Vereinten Nationen mit dieser Parteinahme zu identifizieren. Allerdings gab es weiterhin Fälle, bei denen die Einhegung eines Krieges durch Druck auf alle Kriegsparteien im Vordergrund stand. ${ }^{10}$ Im Sudan wurde ein zunächst asymmetrisches Embargo im März 2005 auf alle Kriegsbeteiligten ausgedehnt.

91995 wurden die Beschränkungen gegenüber der neuen, Tutsi-dominierten Regierung aufgehoben, aber gegenüber Hutu-Rebellen - ohne territoriale Abgrenzung - aufrechterhalten.

10 Ein Faktor, der Einfluss auf das Fehlen asymmetrischer Sanktionen in der Sanktionspraxis der EU hat, ist die Ablehnung von Waffenlieferungen an andere Abnehmer als Regierungen durch die Mitgliedstaaten der EU. Au Grund dieser in allen Mitgliedstaaten verankerten Rechtslage sind Waffenembargos der EU gegen nichtstaatliche Akteure überflüssig.

\section{Kriege ohne Sanktionen}

Sanktionen sind nicht in allen Fällen kriegerischer Auseinandersetzungen ${ }^{11}$ verhängt worden (siehe Tabelle 2). Keine Sanktionen wurden während der Kriege in Uganda, Mozambik, Kongo-Brazzaville, Senegal/Casamance und Guinea-Bissau verhängt. In anderen Fällen gab es Sanktionen von regionalen Staatengruppen, nicht aber von den Vereinten Nationen, wie etwa durch die Nachbarstaaten im Fall von Burundi. Schließlich wurden Sanktionen der Vereinten Nationen häufig relativ lange nach Kriegsbeginn ausgesprochen, während andere Akteure sehr viel früher Sanktionen verhängt hatten, so in den Fällen Angola, Äthiopien/Eritrea, Sudan und DR Kongo.

Zwei Gründe scheinen vor allem für das Fehlen, oder die späte Verhängung, von internationalen Sanktionen relevant gewesen zu sein:

- Geringe internationale Wahrnehmung der Kriege. Die Auseinandersetzungen in Kongo-Brazzaville, Guinea-Bissao, Senegal/ Casamance und auch der Konflikt im Norden Ugandas haben nie besonders große internationale Aufmerksamkeit gefunden. Dies lässt sich zum einen damit erklären, dass die Zahl der Opfer im Vergleich zu anderen Auseinandersetzungen, etwa in der Demokratischen Republik Kongo, deutlich geringer war (wobei der Krieg im Norden Ugandas durch seine lange Dauer inzwischen auch zu den blutigeren Konflikten in Afrika zu zählen ist). Zum anderen war der »CNN-Faktor « nicht im Spiel, keiner der Kriege war Gegenstand einer ausgedehnten Medienberichterstattung, insbesondere nicht in den "globalen Medien « wie den großen Fernsehsendern oder der internationalen Presse ${ }^{12}$. Auch in einer Reihe von Fällen, in denen Sanktionen spät im Verlauf eines Krieges verhängt wurden, wie in Somalia, Liberia oder Ruanda, scheint erst wachsende internationale Aufmerksamkeit notwendig gewesen zu sein, bevor es zu Beschlüssen kam.

- Politik von »Schutzmächten « der Kriegsparteien. Während des Kalten Krieges verhinderten Schutzmächte fast ausnahmslos die Verhängung von Sanktionen. Der Krieg in Mozambique, dessen Wurzeln in die Zeit des Kalten Krieges hineinreichen, ist ein Beispiel hierfür auch noch in den frühen 1990er Jahren. Aber auch nach dem Ende des Kalten Krieges war Widerstand einzelner Staaten, insbesondere solcher mit Vetomacht im VN-Sicherheitsrat für die Verhinderung von Sanktionen ursächlich. So verhinderte Russland eine frühere Verhängung von Sanktionen gegen Äthiopien/Eritrea. Im Fall Sudan waren China und Russland lange gegen Sanktionen, bevor sie dann im Juli 2004 sehr schwachen Sanktionen zustimmten, die im April 2005, nach einem kritischen Bericht des VN-Generalsekretärs über die Lage in Darfur, verschärft wurden.

- Wahrnehmung der Rolle von Sanktionen in der internationalen Politik. Die eingangs geschilderte oft sehr widersprüchliche Einschätzung von Sanktionen, insbesondere von Waffenembargos, spiegelt sich sehr deutlich in der internationalen

11 Für die Tabelle 2 wurde die Kriegsliste von Uppsala/PRIO benutzt, die im Vergleich zu anderen derartigen Listen relativ hohe Hürden für die Aufnahme einer bewaffneten Auseinandersetzung setzt, Ericsson und Wallensteen, a.a.O.

12 Der Konflikt in Nord-Uganda ist eine partielle Ausnahme, wobei die Berichterstattung vor allem über Zwangsrekrutierung von Kindern seit 2004 deutlich zunahm. 
Tabelle 2: Kriege in Afrika südlich der Sahara, internationale Sanktionen und internationale Peacekeeping-Truppen seit 1990

\begin{tabular}{|c|c|c|c|c|}
\hline Land & Krieg* & $\begin{array}{l}\text { Internationale } \\
\text { Sanktionen }\end{array}$ & Sanktionsform & $\begin{array}{l}\text { Internat. Peacekeeping } \\
\text { Truppen }\end{array}$ \\
\hline Angola & $\begin{array}{l}\text { Vor } 1990-1995 \\
1998-2002\end{array}$ & Ab 1993 & Asymmetrisch & Vor 1990-1997 (UNAVEM I-III) \\
\hline Äthiopien & $\begin{array}{l}\text { Vor 1990-1991 } \\
\text { 1998-2001 }\end{array}$ & $1998-2001$ & Symmetrisch & $2000-$ (UNMEE) \\
\hline Burundi & $1997-$ & $\begin{array}{l}1996-2000 \text { (nur } \\
\text { regionale Sanktionen, } \\
\text { nicht international) }\end{array}$ & Symmetrisch & $\begin{array}{l}\text { 2003-2004 (African Union) } \\
\text { 2004-(ONUB) }\end{array}$ \\
\hline DR Kongo & 1996- & $\begin{array}{l}\mathrm{Ab} 1996(\mathrm{EU}) \\
\mathrm{Ab} 2004(\mathrm{VN})\end{array}$ & $\begin{array}{l}\text { Symmetrisch } \\
\text { Asymmetrisch }\end{array}$ & $1999-(\mathrm{MONUC})$ \\
\hline Côte d'Ivoire & $2002-$ & $\mathrm{Ab} 2004$ & Symmetrisch & 2003 (ECOMICI) 2004 - (UNOCI) \\
\hline Eritrea & $\begin{array}{l}\text { Vor 1990-1991 } \\
1998-2001\end{array}$ & $1998-2001$ & Symmetrisch & $2000-(\mathrm{UNMEE})$ \\
\hline Guinea Bissau & 1998-1999 & & & \\
\hline Kongo-Brazzaville & 1997-1999 & & & \\
\hline Liberia & $\begin{array}{l}1990-1995 \\
2002-\end{array}$ & Ab 1992 & Symmetrisch & $\begin{array}{l}\text { 1990-1997 (ECOMOG) } \\
\text { 1993-1997 (UNOMIL) } \\
\text { 2003-(UNMIL) }\end{array}$ \\
\hline Mozambique & Vor 1990-1992 & & & 1992-1994 (ONUMOZ) \\
\hline Ruanda & $\begin{array}{l}1990-1994 \\
1998-2002\end{array}$ & $\mathrm{Ab} 1994$ & $\begin{array}{l}\text { Zunächst symmetrisch, } \\
\text { ab } 1995 \text { asymmetrisch } \\
\text { (Hutu-Rebellen) }\end{array}$ & 1993-1996 (UNAMIR) \\
\hline Senegal/Casamance & $\begin{array}{l}1999-2001, \\
2003\end{array}$ & & & \\
\hline Uganda & $\begin{array}{l}\text { Vor 1990-1991 } \\
1996-2003\end{array}$ & & & \\
\hline Sierra Leone & $1994-2001$ & Ab 1997 & Asymmetrisch & $\begin{array}{l}1997-1999 \\
\text { (ECOMOG/UNOMSIL) } \\
\text { 1999- (UNAMSIL) }\end{array}$ \\
\hline Somalia & Vor $1990-2002$ & seit 1992 & Symmetrisch & 1992-1995 (UNOSOM I, II) \\
\hline Südafrika & Vor 1990-1993 & Bis 1994 & Asymmetrisch & \\
\hline Sudan & Vor 1990- & $\begin{array}{l}\text { Ab } 1996(\mathrm{EU}, \mathrm{VN}) \\
\mathrm{Ab} 2004(\mathrm{VN}) \\
\mathrm{Ab} 2005(\mathrm{VN})\end{array}$ & $\begin{array}{l}\text { Symmetrisch } \\
\text { Asymmetrisch } \\
\text { Symmetrisch }\end{array}$ & \\
\hline
\end{tabular}

Quelle: Uppsala/PRIO Datensatz zu Kriegen, Archiv BICC

* Militärische Auseinandersetzung mit mind. 25 Toten in Kampfhandlungen in einem Jahr und Tausend Toten während des gesamten Krieges.

Sanktionspraxis. Multilaterale Waffenembargos wurden gelegentlich dann verhängt, wenn die internationale Staatengemeinschaft Aktivität zeigen wollte, zu größerem politischen und/oder finanziellen Engagement aber nicht bereit war. Beispiele hierfür sind die Embargos gegen die UNITA in Angola und gegen Ruanda bzw. Huturebellen aus Ruanda sowie das Embargo gegenüber Äthiopien und Eritrea. Das Embargo gegenüber Somalia wurde auch nach Abzug aller VN-Truppen aufrechterhalten. Andererseits wurden in einigen Fällen, in denen internationale Truppen stationiert wurden, keine Waffenembargos verhängt. Dies war z. B. in der DR Kongo der Fall. Auch in Burundi gibt es seit 2003 Peacekeeping ohne Waffenembargo.

\section{Die Wirkung von Sanktionen}

In der Beurteilung der Sinnhaftigkeit von Sanktionen steht die Frage nach deren Effektivität oft im Vordergrund, ohne dass allerdings immer klar ist, was damit gemeint ist, und wie sie gemessen werden soll. Effektivität, allgemein definiert als Grad der Zielerreichung, erfordert die Bestimmung der Ziele von Sanktionen.

Grob lassen sich zwei Arten von Zielen von Sanktionen unterscheiden. Die erste Art von Zielen betrifft die Umsetzung der Sanktionen. Hier geht es darum, inwieweit die Sanktionsbeschlüsse internationaler Organisationen befolgt werden und ob es Schlupflöcher und Ausweichmöglichkeiten für die Sanktionierten gibt (Sanktionsziel). Die zweite Art von Zielen 
Tabelle 3: Effektivität von internationalen Sanktionen in Afrika seit 1990

\begin{tabular}{|c|c|c|c|}
\hline Ziel & Sanktionsziel & Politikziel & Anmerkungen \\
\hline UNITA in Angola & $\begin{array}{l}\text { Über längeren Zeitraum, auch } \\
\text { durch Einführung weiterer } \\
\text { Sanktionstypen weitgehend } \\
\text { erreicht }\end{array}$ & $\begin{array}{l}\text { Politikziel indirekt } \\
\text { erreicht, auch durch } \\
\text { Sanktionen }\end{array}$ & $\begin{array}{l}\text { Verstärkung der Asymmetrie durch } \\
\text { Sanktionen; Zentral: Verknüpfung } \\
\text { verschiedener Sanktionstypen }\end{array}$ \\
\hline Äthiopien & Erreicht & $\begin{array}{l}\text { Wahrscheinlich kein } \\
\text { Einfluss }\end{array}$ & $\begin{array}{l}\text { Keine bekannten Waffenlieferungen } \\
\text { während Sanktionen }\end{array}$ \\
\hline Burundi & $\begin{array}{l}\text { Teilweise erreicht (starke } \\
\text { Erhöhung von Preisen) }\end{array}$ & Teilweise erreicht & $\begin{array}{l}\text { Friedensverhandlungen, aber nicht } \\
\text { mit allen relevanten Gruppen }\end{array}$ \\
\hline $\begin{array}{l}\text { Demokratische Republik } \\
\text { Kongo (Zaire) }\end{array}$ & Nicht erreicht & Nicht erreicht & $\begin{array}{l}\text { Möglicherweise Verbesserung durch } \\
\text { Mandat MONUC }\end{array}$ \\
\hline Côte d'Ivoire & Teilweise erreicht & $\begin{array}{l}\text { Teilweise erreicht } \\
\text { (Wiederaufnahme des } \\
\text { Friedensprozesses) }\end{array}$ & $\begin{array}{l}\text { Friedensprozesses läuft mit } \\
\text { erheblichen Schwierigkeiten }\end{array}$ \\
\hline Eritrea & Erreicht & $\begin{array}{l}\text { Wahrscheinlich kein } \\
\text { Einfluss }\end{array}$ & $\begin{array}{l}\text { Keine bekannten Waffenlieferungen } \\
\text { während Sanktionen }\end{array}$ \\
\hline Liberia & $\begin{array}{l}\text { Im Zeitablauf verbesserte } \\
\text { Umsetzung, aber trotz } \\
\text { Erweiterung der Sanktionen } \\
\text { mangelhaft }\end{array}$ & $\begin{array}{l}\text { Ziel erreicht, teilweise } \\
\text { auch durch Sanktionen }\end{array}$ & $\begin{array}{l}\text { Militärische Schwäche wesentlicher } \\
\text { Faktor für Verhandlungsbereitschaft } \\
\text { Taylor-Regierung }\end{array}$ \\
\hline $\begin{array}{l}\text { RUF, Militärregierung in } \\
\text { Sierra Leone }\end{array}$ & $\begin{array}{l}\text { Im Zeitablauf verbesserte } \\
\text { Umsetzung aber immer mit } \\
\text { Problemen behaftet }\end{array}$ & $\begin{array}{l}\text { Ziel erreicht, auch durch } \\
\text { Sanktionen }\end{array}$ & $\begin{array}{l}\text { Schwächung RUF/AFRC ein } \\
\text { Element der Verhandlungs- } \\
\text { bereitschaft }\end{array}$ \\
\hline $\begin{array}{l}\text { Ruanda, Regierung } \\
\text { später Hutu-Rebellen in } \\
\text { Nachbarstaaten }\end{array}$ & Nur in geringem Masse erreicht & $\begin{array}{l}\text { Erste Phase erreicht } \\
\text { (Völkermord und } \\
\text { Bürgerkrieg beendet) aber } \\
\text { Sanktionen wahrscheinlich } \\
\text { kein Einfluss; 2. Phase: } \\
\text { nicht erreicht (Hutu } \\
\text { Milizen weiter aktiv) }\end{array}$ & $\begin{array}{l}\text { Waffenlieferungen durch Embargo } \\
\text { nur geringfügig beeinträchtigt }\end{array}$ \\
\hline Somalia & Nicht erreicht & Nicht erreicht & \\
\hline Sudan & Ab 1996: Nicht erreicht & Nicht erreicht & $\begin{array}{l}\text { Ausweitung des Embargos auf } \\
\text { sudanesische Regierung angedroht }\end{array}$ \\
\hline Südafrika & $\begin{array}{l}\text { Teilweise erreicht, Beitrag } \\
\text { möglich }\end{array}$ & $\begin{array}{l}\text { Politikziel nach 14 Jahren } \\
\text { erreicht }\end{array}$ & $\begin{array}{l}\text { Schwächung der Wirtschaft } \\
\text { (nationale Sanktionen) und der } \\
\text { Streitkräfte ein Element der } \\
\text { Verhandlungsbereitschaft }\end{array}$ \\
\hline Togo & $\begin{array}{l}\text { Weitgehend erreicht (über sehr } \\
\text { kurze Zeitspanne) }\end{array}$ & $\begin{array}{l}\text { Weitgehend erreicht } \\
\text { (Wahlen, aber mit } \\
\text { umstrittenen Ausgang) }\end{array}$ & $\begin{array}{l}\text { Effekt der Sanktionen als Symbole } \\
\text { ausländischer Ablehnung des } \\
\text { Machtwechsels, nicht direkt }\end{array}$ \\
\hline
\end{tabular}

betrifft die Änderung des inkriminierten Verhaltens, Fragen nach dem Umfang der Änderung des Verhaltens und des Einflusses der Sanktionen darauf (Politikziel).

Die Wirkungen der Sanktionen in Afrika sind in Tabelle 3 beschrieben. Daraus geht hervor:

- In vier Fällen wurden Sanktionsziele weitgehend erreicht. Allerdings hatte dies nur in einem Fall auch merkliche Auswirkungen auf die Politikziele (Angola), in drei Fällen war dies nicht der Fall (Äthiopien, Eritrea, Togo).

- In fünf Fällen wurden die Sanktionsziele teilweise erreicht. Die Mengen an importierten Waffen gingen deutlich zurück, die Preise für Waffenimporte stiegen merklich. In diesen fünf
Fällen (Burundi, Côte d'Ivoire, Liberia, Sierra Leone und Südafrika) wurden auch die Politikziele zumindest teilweise erreicht, wobei nicht klar ist, ob Sanktionen dabei eine wichtige Rolle spielten.

- In vier Fällen wurden die Sanktionsziele nicht erreicht (Kongo, Ruanda, Somalia, Sudan).

- In den meisten Fällen, in denen Sanktionsziele und Politikziele erreicht wurden, wurden mehrere Sanktionstypen miteinander kombiniert (Liberia, Sierra Leone, Côte d'Ivoire) bzw. umfassende Sanktionen umgesetzt (Burundi). In den Fällen, in denen weder bei Sanktions- noch bei Politikzielen Erfolge zu verzeichnen sind, handelt es sich in der Regel um allein stehende Waffenembargos. 
Tabelle 4: Bewaffnete nicht-staatliche Gruppen in der ECOWAS-Region und ihre Bezugsquellen für Waffen

\begin{tabular}{|c|c|c|}
\hline Land & Gruppe & Quelle für Waffen/Unterstützung \\
\hline Côte d'Ivoire & $\begin{array}{l}\text { MPCI } \\
\text { MPIGO } \\
\text { MJP } \\
\text { Lima } \\
\text { GPP/CPP/FLN } \\
\text { FSCO } \\
\text { FLGO } \\
\text { Young Patriots }\end{array}$ & $\begin{array}{l}\text { Burkina Faso?, Ivorische Streitkräfte (Diebstahl) } \\
\text { Liberia (Taylor) } \\
\text { Liberia (Taylor) } \\
\text { Ivorische Streitkräfte, Liberia (MODEL) } \\
\text { Ivorische Streitkräfte } \\
\text { Ivorische Streitkräfte } \\
\text { Ivorische Streitkräfte } \\
\text { Ivorische Streitkräfte }\end{array}$ \\
\hline Guinea & $\begin{array}{l}\text { RFDG } \\
\text { Young Volunteers }\end{array}$ & $\begin{array}{l}\text { Liberia (Taylor), Sierra Leone (RUF) } \\
\text { Guineische Streitkräfte }\end{array}$ \\
\hline Guinea-Bissao & MFDC (ex-Mané Anhänger) & Streitkräfte Guinea-Bissaos (Diebstahl) \\
\hline Liberia & $\begin{array}{l}\text { LURD } \\
\text { MODEL } \\
\text { NPFL (Taylor) }\end{array}$ & $\begin{array}{l}\text { Guinea (Regierung), Sierra Leone (diverse Gruppen) } \\
\text { Côte d'Ivoire (Regierung) } \\
\text { Osteuropa (Bulgarien, Ukraine) über Burkina Faso, Ostasien }\end{array}$ \\
\hline Niger & Tuareg and Tobou groups & Lokaler Waffenhandel, Diebstahl aus Regierungsarsenalen \\
\hline Nigeria & $\begin{array}{l}\text { Bakassi Boys/VS } \\
\text { OPC } \\
\text { APC } \\
\text { EBA } \\
\text { NDVF } \\
\text { MASSOB } \\
\text { FNDIC } \\
\text { Al-Sunna Wal Jamma }\end{array}$ & $\begin{array}{l}\text { Region } \\
\text { Region } \\
\text { Nigerianische Streitkräfte (ehemalige Angehörige) } \\
\text { Region } \\
? \\
\text { Region } \\
\text { ? } \\
\text { Saudi Arabien? }\end{array}$ \\
\hline Senegal & MFDC & Guinea-Bissao (bis 1998), lokaler Waffenhandel \\
\hline Sierra Leone & $\begin{array}{l}\text { RUF } \\
\text { CDF/Kamajor }\end{array}$ & $\begin{array}{l}\text { Liberia (Taylor), Osteuropa (Bulgarien, Ukraine) über Côte } \\
\text { d'Ivoire, Burkina Faso } \\
\text { Streitkräfte, ECOMOG }\end{array}$ \\
\hline
\end{tabular}

Quelle: Nicolas Florquin und Eric G. Berman (Hrsg.), Armed and Aimless: Armed Groups, Guns, and Human Security in the ECOWAS Region, Genf, 2005, Part II.

- Der Nachschub an schweren Waffen konnte vermindert werden (Südafrika, Äthiopien/Eritrea), während der Nachschub an kleinen und leichten Waffen sowie Munition kaum vermindert werden konnte.

\section{Lehren aus der Sanktionspraxis in Afrika}

Internationale Sanktionspolitik und -praxis der letzten Jahrzehnte weisen kein einheitliches Muster auf. Weitgehend wirkungslosen Waffenembargos stehen Fälle gegenüber, in denen Sanktionen Auswirkungen in die gewünschte Richtung gehabt haben. Einige Trends lassen sich erkennen:

- Waffenembargos sind im Laufe der 1990er Jahre in Bezug auf das Sanktionsziel effektiver geworden. Das heißt weder, dass der Erfolg durchschlagend war, noch dass dadurch auch die Politikziele erreicht wurden. Dennoch zeigen die Daten im Untersuchungszeitraum eine leicht ansteigende Zahl von Fällen, in denen Waffenembargos Wirkungen hatten.
- Ein Grund für diesen tendenziell zunehmenden Erfolg von Waffenembargos ist die Entwicklung von »intelligenten Sanktionen « in zumindest einigen Fällen, wie Angola, Sierra Leone und Liberia. Die Ende der 1990er Jahre bei den Vereinten Nationen initiierte Sanktionsreform hat zumindest in einigen Fällen zu einem differenzierteren Umgang mit diesem Instrument internationaler Politik geführt, der auch einige Erfolge gezeitigt hat.

- Ein wesentliches Element der Sanktionsreform ist die Suche nach und Kombination von Sanktionen, von denen erwartet wird, dass sie den Sanktionierten gezielt treffen, Unbeteiligte hingegen weitgehend schonen ${ }^{13}$. Insbesondere Finanzsanktionen und selektive Beschränkungen des Exports haben sich als wirkungsvolle Elemente umfassender gezielter Sanktionspakete erwiesen. Reisebeschränkungen und Im-

13 David Cortright und George Lopez, Smart Sanctions. Targeting Economic Statecraft, Lanham, 2002; Michael Brzoska, From dumb to smart? Recent sanctions reform at the United Nations, Global Governance, 9 (4), 2003. 
portsanktionen für Öl trugen in einigen Fällen ebenfalls zur Wirkung von Sanktionspaketen bei.

- Die Mitglieder des Sicherheitsrates der Vereinten Nationen haben sich mit Sanktionen relativ häufig auf die Seite einer Kriegspartei geschlagen (asymmetrische Sanktionen). Durch solche Parteinahme stieg die Wahrscheinlichkeit des Erfolges von Sanktionen.

- Ein anderes Element, das zu einer tendenziell zunehmenden Wirksamkeit von Waffenembargos beitrug, sind die internationalen Bemühungen um die Kontrolle von Klein- und Leichtwaffen $^{14}$. Der Grad der Verfügbarkeit von Waffen in Afrika ist weiterhin hoch. Allerdings hat sich der Charakter des Nachschubs von Waffen und Munition in Krisenund Kriegsregionen in den letzten Jahren stark verändert. Während in den 1990er Jahren die Waffen vornehmlich aus Osteuropa kamen und von privaten Waffenhändlern vermittelt wurden, die auch den Transport in die Krisenund Kriegsgebiete Afrikas in der Hand hatten, fließen diese Quellen zunehmend spärlicher. Länder wie Bulgarien und, mit Abstrichen, auch Serbien und die Ukraine, haben ihre Waffenexportkontrollen verbessert. Die bekanntesten Waffenhändler, wie Victor Bout und Alexander Minin, haben ihre Geschäftstätigkeit auf andere Felder verlagert.

- Der Nachschub von Waffen und Munition ist in Afrika wieder stärker »verstaatlicht « worden. Rebellen werden in Afrika gegenwärtig vor allem von Regierungen aus Nachbarländern versorgt. Das lässt sich etwa an Hand der wichtigsten Rebellenbewegungen in Westafrika zeigen (Tabelle 4).

- Sehr widersprüchlich ist das Verhältnis von Sanktionen, insbesondere Waffenembargos, und dem Einsatz multilateraler Truppen. So hatten internationale Missionen in der Vergangenheit kein Mandat, Waffenembargos zu überwachen, selbst in den Fällen, in denen ein solches Waffenembargo von den Vereinten Nationen verhängt worden war. Der erste Fall, in denen eine VN-Mission ausdrücklich auch mit der Überwachung eines Waffenembargos betraut wurde, ist der der DR Kongo, danach folgte Côte d'Ivoire. Fast zeitgleich hat der VN-Sicherheitsrat im Frühjahr 2004 im Fall Burundi einen internationalen Truppeneinsatz beschlossen, ohne dass es ein Waffenembargo gibt. Eine bessere Koordination von Sanktionen, insbesondere von Waffenembargos und internationalen Truppeneinsätzen, scheint dringend geboten.

\section{Ausblick}

Sanktionen, vornehmlich Waffenembargos, sind seit den 1990er Jahren häufig als Instrument zur Einhegung von Konflikten in Afrika eingesetzt worden, trotz einer insgesamt wenig überzeugenden Erfolgsbilanz. Obwohl eine Reihe von Reformmaßnahmen, insbesondere bei den VN selber, eingeleitet worden sind, bleiben wesentliche Mängel der Umsetzung bestehen. Waffenembargos und andere Sanktionen werden weiter hinter den, oft sehr hohen, Erwartungen hinterherhinken, wenn die wichtigsten Akteure, nämlich die Staaten, aus denen Waffen geliefert werden, und die regionalen Nachbarn

14 Small Arms Survey, Oxford, 2004. der sanktionierten Staaten und Gruppen, über deren Territorien die Waffen und andere sanktionierte Güter fließen, sie nicht ernsthafter und mit größerem Einsatz von Kontrollinstrumenten implementieren. Auch im besten Fall allerdings können Waffenembargos nur einen Beitrag zur Verhinderung und Eingrenzung von Konflikten leisten, isoliert sind sie dazu in aller Regel nicht geeignet. Waffenembargos sind sinnvoll, wenn sie in umfassende politische Strategien des Konfliktmanagements eingebettet sind. Umgekehrt sind Ansätze des Krisenmanagements problematisch, wenn sie nicht von Waffenembargos begleitet werden.

In den letzten Jahren haben sich, nicht zuletzt auch wegen der verstärkten Diskussion über die Umsetzung von Waffenembargos, die Exportpolitiken einer Reihe von früher besonders problematischen Exporteurstaaten verbessert. Beispiele dafür sind vor allem ost- und südosteuropäische Länder, die seit 2004 Mitglieder der Europäischen Union sind oder die diese Mitgliedschaft anstreben. Obwohl weiterhin Lücken in der Kontrolle von Exporteuren bestehen, etwa in der Ukraine oder in Moldawien, kommt die überwiegende Menge des Nachschubs an Waffen und Munition in afrikanischen Kriegen gegenwärtig vermutlich aus Nachbarstaaten.

Für eine weitere Verbesserung von Sanktionspolitik und -praxis in Afrika scheint deshalb ein verstärktes Engagement von Regionalorganisationen besonders hilfreich. Bisher haben Regionalorganisationen nur eine untergeordnete Rolle bei der Formulierung und Umsetzung von Sanktionen gehabt. So haben inzwischen praktisch alle Regionalorganisationen mehr oder weniger effektive Kleinwaffenprogramme. Regionalorganisationen könnten vor allem drei Aufgaben übernehmen:

- Die Beratung der Vereinten Nationen bei der Entscheidungsfindung über Sanktionen. Immer noch werden Sanktionen ohne gründliche Analyse ihrer wahrscheinlichen Wirkungen verhängt. Die VN selber haben nur begrenzte Möglichkeiten eigenständiger Analyse; vielen Mitgliedstaaten des VN-Sicherheitsrates geht es genauso. Selbst die großen Mitgliedstaaten verfügen oft nur über unzureichende Informationen, insbesondere wenn ein Konflikt neu ins Blickfeld gerät oder Großmachtinteressen nicht betroffen sind.

- Praktische Unterstützung von Nachbarstaaten eines sanktionierten Landes bei der Umsetzung von Sanktionen. Beispiele sind die Stellung von Grenzpolizisten und Hilfe bei der Überwachung des Luftraumes. Diese Unterstützung wird, aus finanziellen Gründen, ohne die westlichen Staaten kaum möglich sein, könnte aber regional organisiert werden. Ein Beispiel aus Europa für derartige Hilfe bei der Umsetzung von Sanktionen ist die Sanctions Assistance Mission (SAM) der Westeuropäischen Union während des Krieges im ehemaligen Jugoslawien ${ }^{15}$.

- Ausbildung von Beamten, in Ministerien, bei Zoll und Polizei, in Nachbarländern des sanktionierten Landes, zur Verbesserung und Vereinheitlichung der Sanktionspraxis. 